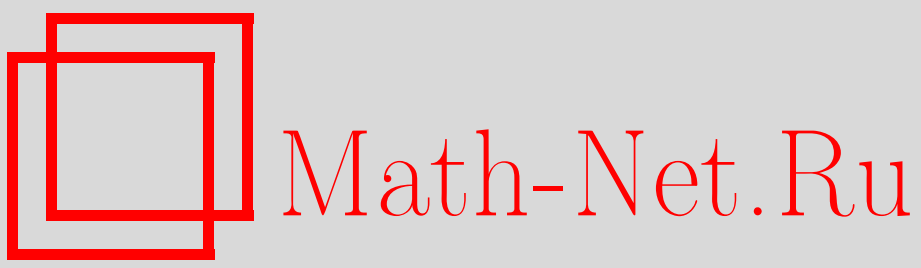

О. М. Минеева, Об устойчивости решения одной краевой задачи гидродинамики, Матем. заметки, 1996, том 59, выпуск 5, 774-776

DOI: https://doi.org/10.4213/mzm1773

Использование Общероссийского математического портала MathNet.Ru подразумевает, что вы прочитали и согласны с пользовательским соглашением

http://www. mathnet.ru/rus/agreement

Параметры загрузки:

IP : 52.90 .164 .192

26 апреля 2023 г., 06:02:44

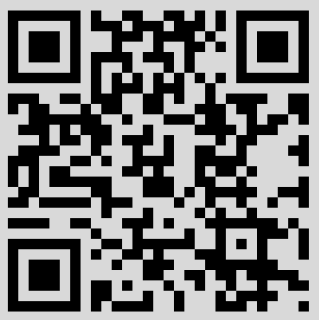




\section{ОБ УСТОЙЧИВОСТИ РЕШЕНИЯ ОДНОЙ КРАЕВОЙ ЗАДАЧИ ГИДРОДИНАМИКИ}

\section{О. М. Минеева}

Уравнение

$$
\begin{aligned}
\frac{\partial^{2} u}{\partial t^{2}}+\left(\alpha \frac{\partial^{5} u}{\partial x^{4} \partial t}+k \frac{\partial u}{\partial t}\right. & \left.+2 \beta U(t) \frac{\partial^{2} u}{\partial x \partial t}\right) \\
+ & \left(\frac{\partial^{4} u}{\partial x^{4}}+U^{2}(t) \frac{\partial^{2} u}{\partial x^{2}}+\beta \frac{\partial^{2} U(t)}{\partial t} \frac{\partial u}{\partial x}\right)=0
\end{aligned}
$$

с краевыми условиями

$$
u(0, t)=u(1, t)=\frac{\partial u}{\partial x}(0, t)=\frac{\partial u}{\partial x}(1, t)=0
$$

описывает колебания трубопровода с закрепленными концами, находящегося в вязкой среде и несущего поток несжимаемой жидкости [1].

При постоянной скорости вытекающей жидкости $U(t)$, устойчивость нулевого решения была исследована в [2]-[6], а для случая, когда эта скорость имеет вид $U(t)=v_{0}(1+\sigma \cos \omega t), \sigma>0, \omega>0$, задача $(1),(2)$ была исследована в [1], [7]. В работе [1] получены формулы, описьвающие область неустойчивости с точностью до малых величин. В работе [7] доказано, что устойчивость нулевого решения будет иметь место при $v_{0} \in[0,2 \pi]$ для каждого $\omega>0$ и $\sigma \in\left(0, \sigma_{0}\right), \sigma_{0}=\sigma_{0}\left(v_{0}, \omega\right)$ без указания конкретного вида $\sigma\left(v_{0}, \omega\right)$.

Цель настоящей работы - установление абстрактного результата, позволяющего исследовать устойчивость нулевого решения задачи $(1),(2)$ в зависимости от скорости вытекающей жидкости $U(t)$.

Рассмотрим в гильбертовом пространстве $H$ операторное уравнение

$$
\ddot{u}(t)\left(B_{1}(t)+B_{2}(t)\right) \dot{u}(t)+\left(A_{1}(t)+A_{2}(t)\right) u(t)=0
$$

с начальными условиями

$$
u(0)=u_{0}, \quad \dot{u}(0)=u_{1} .
$$

Относительно операторов $A_{1}(t), A_{2}(t), B_{1}(t), B_{2}(t)$ будем предполагать, что они определены на некоторой постоянной области определения $D_{0} \subset H$ и обладают следующими свойствами:

1) $A_{1}(t)$ самосопряжен, сильно непрерьвен по $t, B_{1}(t)$ - симметрический, $A_{2}(t), B_{2}(t)$ - кососимметрические;

2) $\exists p>0$ такое, что $\forall t\left|A_{1}(t)\right| \geqslant p I$;

3) $\exists b_{1}>0$ такое, что $\forall t B_{1}(t) \geqslant b_{1} I$;

4) пусть $\forall t \quad D_{0} \subset D_{t}^{\prime}$, где $D_{t}^{\prime}$ - область определения оператора $\dot{A}(t)$,

$$
\dot{A}(t) v=s-\lim _{\Delta t \rightarrow 0} \frac{A(t+\Delta t)-A(t)}{\Delta t} v .
$$

Решением задачи $(3),(4)$ будем назьвать дважды дифференцируемую функцию $u(t)$ такую, что при каждом $t: u(t) \in D_{0}, \dot{u}(t) \in D\left(A_{1}^{1 / 2}\right)$, удовлетворяющую (3), (4). В пространстве дифференцируемых функций со значениями в $D_{0}$ рассмотрим 
норму $\|u(t)\|_{1}=\sqrt{(\dot{u}(t), \dot{u}(t))+(|A(t)| u(t), u(t))}$. Нулевое решение $(3),(4)$ назовем устойчивым, если существует такая константа $C>0$, что для любого решения $u(t)$ задачи $(3),(4)$ выполнено неравенство $\|u(t)\|_{1} \leqslant C\|u(0)\|_{1}$. В противном случае, т.е. если для любой константы $C>0$ найдется такое решение $(3),(4)$, что при некотором $t_{0}$ имеет место: $\left\|u\left(t_{0}\right)\right\|_{1} \geqslant C\|u(0)\|_{1}$, нулевое решение $(3),(4)$ будем назьвать неустойчивым.

В случае, когда при некоторых константах $C, k$ любое решение $(3),(4)$ удовлетворяет неравенству $\|u(t)\|_{1} \leqslant C e^{-k t}\|u(0)\|_{1}$, нулевое решение будем назьвать экспоненциально устойчивым.

Справедлива следующая теорема.

ТЕОРемА. Пусть найдутся строго полохительнье числа $\eta, \rho, \theta, s u$ $\varepsilon<1 / \sqrt{p}$, удовлетворяющ,ие неравенству

$$
b_{1} \rho+2 \varepsilon+\theta+\eta+s \leqslant 2 b_{1}
$$

такие, что

$$
\dot{A}_{1}(t) \leqslant(2 \varepsilon+s) A_{1}(t)+\frac{1}{\theta} A_{2}^{2}(t)-\frac{\varepsilon^{2}}{\rho} B_{1}(t)+\frac{\varepsilon^{2}}{\eta} B_{2}^{2}(t) .
$$

Тогда если оператор $A_{1}(t)$ полохителен при всех значениях $t>0$, то нулевое решение задачи (3), (4) әкспоненциально устойчиво; если же найдется әлемент $\varphi_{0} \in D_{0}$ такой, что $\left(A_{1}(0) \varphi_{0}, \varphi_{0}\right)<0$, то нулевое решение неустойчиво. Если же найдутся строго положительные числа $\eta, \rho, \theta$ $u \varepsilon<1 / \sqrt{p}$ такие, что неравенства (5), (6) выполнены при $s=0$, то в предположсени $A_{1}(t)>0$ для всех $t>0$ нулевое решение (3), (4) является устойчивым (но, возмохсно, не экспоненииально устойчивым).

Эта теорема следующим образом может быть применена к задаче (1), (2).

В пространстве дважды дифференцируемых по $t$ функций со значениями в пространстве Соболева $W_{4}^{2}(0,1) x$ рассмотрим энергетическую норму

$$
\|u(\cdot, t)\|_{1}=\sqrt{(\dot{u}(\cdot, t), \dot{u}(\cdot, t))+\left(A_{1}(t) u(\cdot, t), u(\cdot, t)\right)},
$$

где

$$
\begin{gathered}
A_{1}(t) u=\frac{\partial^{4} u}{\partial x^{4}}+U^{2}(t) \frac{\partial^{2} u}{\partial x^{2}} \\
D\left(A_{1}(t)\right)=\left\{u \in W_{4}^{2}(0,1) \mid u(0)=u(1)=\frac{\partial u(0)}{\partial x}=\frac{\partial u(1)}{\partial x}\right\} .
\end{gathered}
$$

Положим также

$$
\begin{gathered}
A_{2}(t) u=\frac{\partial U}{\partial t} \beta \frac{\partial u}{\partial x}, \quad D\left(A_{2}(t)\right)=D_{0} \\
B_{1} u=\alpha \frac{\partial^{4} u}{\partial x^{4}}+k u, \quad D\left(B_{1}\right)=D_{0} \\
B_{2}(t) u=2 \beta U(t) \frac{\partial u}{\partial t}, \quad D\left(B_{2}(t)\right)=D_{0} .
\end{gathered}
$$

Тогда справедливы следующие утверждения.

СЛЕДСтвИЕ 1. Если скорость вытекающей жсидкости $U(t)$ монотонна, выпукла вверх и

$$
\frac{\beta^{2}}{4(\alpha \Theta+k)} \dot{U}(0)<U(0),
$$


әде $\Theta$ - наименьший положительныи корень уравнения $\cos x \cdot\left(e^{x}-e^{-x}\right) / 2=1$, то в случае, когда $\forall t \quad U(t) \in(0,2 \pi)$, нулевое решение әкспоненциально устойчиво. В случае, когда $\forall t \quad U(t) \in\left(d_{i}, d_{i+1}\right)$ при некотором $i$, где $d_{i}-$ расположенные в порядке возрастания положстельные корни уравнения $\sin \frac{x}{2}\left(\operatorname{tg} \frac{x}{2}-\frac{x}{2}\right)=0$, то нулевое решение неустойчиво.

СЛЕДСТВИЕ 2. Если скорость вытекающей жсидкости $U(t)$ монотонно убивающая, вогнутая $u \forall t \quad U(t)<2 \pi$, то при достаточно мальх $U(0), \dot{U}(0)$ нулевое решение будет әкспоненциально устойчиво. А именно, $U(0)$ и $\dot{U}(0)$ долхны быть настолько малы, чтобы при некотором

$$
\varepsilon<\min \left(k+\Theta \alpha ; \frac{1}{\sqrt{\Theta\left(1-(U(0) / 2 \pi)^{2}\right)}}\right)
$$

выполнялось неравенство

$$
\frac{\left(\varepsilon\left(\beta V(0)-\frac{1}{2}\left(\frac{k}{\Theta}+\alpha\right) \sqrt{\Theta}\right)-\frac{1}{2} \dot{V}(0) \beta\right)^{2}}{(\alpha \Theta+k)-\varepsilon}+\varepsilon\left(V^{2}(0)-1\right)-V(0) \dot{V}(0)<0,
$$

әде $V(t)=U(t) /(2 \pi), \Theta-$ то же, что и в следствии 1 .

СлЕдСтВИЕ 3. Если скорость вытекающей жсидкости имеет вид $U(t)=v_{0}(1+\sigma \cos \omega t)$, m.е. периодична, тогда для всех полохительньх $v_{0}<2 \pi \quad u \quad \sigma<1$ maкux, что $v_{0}(1+\sigma)<2 \pi$ найдется $\omega_{0}=\omega_{0}(\sigma)$ такое, что для всех $\omega \in\left(0, \omega_{0}\right)$ нулевое решение будет әкспоненциально устойчиво. При этом $\omega_{0}$ определяется следующим образом. Обозначим

$$
\begin{gathered}
\varepsilon^{*}=\min \left(k+\Theta \alpha ; \frac{1}{\sqrt{\Theta\left(1-b^{2}(1+\sigma)^{2}\right)}}\right) ; \quad m_{1}=b \beta \sigma, \quad m_{2}=b^{2}(1+\sigma) \sigma, \\
m_{3}=1-b(1+\sigma), \quad m_{4}=\alpha \Theta+k, \quad m_{5}=b \beta(1+\sigma)+\frac{1}{2}\left(\frac{k}{\Theta}+\alpha\right) \sqrt{\Theta} .
\end{gathered}
$$

Тогда

$$
\begin{array}{r}
\omega_{0}=\max _{\varepsilon \leqslant \varepsilon^{*}} \frac{1}{m_{1}}\left[\sqrt{\left(m_{2} m_{4}+\varepsilon\left(m_{1} m_{5}-m_{2}\right)\right)^{2} 2 m_{1}\left(\varepsilon m_{3} m_{4}-\varepsilon^{2}\left(m_{3}+m_{5}\right)\right)}\right. \\
\left.-m_{2} m_{4}-\varepsilon\left(m_{1} m_{5}-m_{2}\right)\right] .
\end{array}
$$

Поступило

27.11 .95

\section{СПИСОК ЦИТИРОВАННОЙ ЛИТЕРАТУРЫ}

1. Челомей С. В. // Изв. АН СССР. Сер. мех. тв. тела. 1984. № 5. С. 170-174. 2. Феодосьев В. Н. // Инж. сб. 1951. Т. 10. С. 169-170. 3. Мовчан А. А. // ПММ. 1965. Т. 29. № 4. С. 760-762. 4. Доценко П. Д. // Прикл. механика. 1979. Т. 15. №1. С. 69-75. 5. Зефиров В. И., Колесов В. В., Милославский А. И. // Изв. АН СССР. Сер. мех. тв. тела. 1985. №1. С. 179-187. 6. Милославский А. И., Станиславский Ю. Л . // Изв. АН СССР. Сер. мех. тв. тела. 1988. № 5. С. 160-167. 7. Буй Та Лонг. Применение функциональных методов к некоторым задачам устойчивости. Дисс. ... к. ф.-м. н. М.: МГУ, 1988. 